Received: October 12, 2017

Revision received: May 2, 2018

Research Article

\title{
Research on Teacher-Student Interaction in M-learning*
}

\author{
Xiaheng Zhang ${ }^{1}$ \\ Northwest University of Political Science \\ and Low
}

\author{
Dan Wang ${ }^{2}$ \\ Northwest University of Political \\ Science and Low
}

\begin{abstract}
This paper attempts to thoroughly explore the teacher-student interaction in the context of m-learning. To this end, the service capacity parameters and influencing factors of m-learning platform were introduced, and a game income matrix of the teacher-student interaction was created to yield the Nash equilibrium solution. It is found that the service capacity of m-learning platform is negatively correlated with the probability of teacher supervision, the supervision cost is negatively correlated with the probability of students' active cooperation, and the penalty amount imposed on the teacher is positively correlated with the probability of students' active cooperation. The research findings fill up the research gap on the teacher-student interaction in m-learning.
\end{abstract}

\section{Keywords}

M-learning • Teacher-Student Interaction • Game Theory

\footnotetext{
* Supported by the National Civil Affairs Commission's ethnic research project "Research on the development of cross-border e-commerce in the northwest ethnic areas of the silk road economic belt" (2018-GMD-021), and the Young Academic Innovation Team of Northwest University of Political Science and Law.

${ }^{1}$ Correspondence to: Xiaheng Zhang (PhD), Business School, Northwest University of Political Science and Law, Xi'an 710122, China. Email: zhangxiaheng@163.com

${ }^{2}$ Business School, Northwest University of Political Science and Law, Xi’an 710122, China. Email: wangdanpoiu@qq.com
} 
The interaction between the teacher and students (teacher-student interaction) is the most basic and common form of interaction in the course of teaching. As an important support to student learning, the teacher-student interaction greatly bolsters the development of students. Besides clarifying the learning method, the interaction provides students with loads of learning opportunities, and stands out as the key to student growth (Bernstein \& Noam, 2013). In classroom teaching, the teacher-student interaction is a critical social process for students to achieve academic, social, emotional development and peer interaction (Rutter \& Maughan, 2002).

With the proliferation of smartphones and tablet computers, mankind has entered an information age with the mobile Internet as the medium of communication. In the field of education, the new trend is manifested in the form of m-learning. The promotion of m-learning has reshaped the traditional face-to-face teacher-student interaction. Thanks to the mobile Internet, the education elements in the interaction are expressed in a brandnew form. The teacher now imparts knowledge, exchanges ideas, shares values and learns together with students in cyberspace.

The mobile Internet shortens the cycle knowledge updating, fragmentize the knowledge acquisition process, and transforms the learning and teaching modes. The traditional face-to-face teacher-student interaction has been severely impacted by the mobile Internet in every aspect. As mentioned before, the teacher-student interaction is the most fundamental and prevalent form of interaction in teaching. A good interaction benefits the development of both the teacher and the students, and elevate the overall education quality. With m-learning being a research hotspot, it is both theoretically and practically meaningful to explore teacher-student interaction in the context of m-learning.

\section{Literature Review}

The research on the Internet-based teacher-student interaction is rather limited. The few studies are concentrated on the relationship between the teacher and students. The previous works mainly focus on the following three aspects.

First, the nature and features of the Internet-based teacher-student relationship. Ma et al., held that the teacher and students are truly equal in the Internet-based relationship (2010). Second, the carrier of teacherstudent interaction in the Internet. Wan et al., discussed the social interaction of e-learning community based on QQ groups (2012). Liu et al., explored the mobile teaching on the SMS interactive platform (2011). Third, the influencing factors of the Internet-based teacher-student relationship. Through experimental analysis, Zhang $\&$ Zuo, discovered that the teacher-student interaction is influenced by the interactive environment, personal traits, events of different emotional valences, and the emotional state of students (2013).

Despite the increasing popularity of m-learning, the teacher-student interaction has not been properly adjusted in reality. The static interaction results are still being emphasized over the structure and relationship of the interaction process. This rigid view of learning now restricts the effect of the teacher-student interaction. The existing research has not tackled the teacher-student interaction in m-learning, not to mention giving sound solutions to the three aspects of Internet-based relationship above. 
The relationship between the two subjects of m-learning, the teacher and students, is both cooperative and conflictive in nature. Inspired by the traditional bipolar cooperation model, this paper analyses the teacherstudent interaction based on the static game model with complete information.

\section{Modelling of teacher-student interaction}

\section{Hypotheses}

In an m-learning environment, suppose there is a teacher A and a class of students B. Unlike traditional learning, $\mathrm{B}$ is in the dominant position of the teacher-student relationship, to which A provides the required teaching services. In return, B pays a fee to A for the services. Being a cost to B and an income to A, the fee consists of two parts, namely, the fixed cost and the performance cost. The fixed cost $\mathrm{W}$ is the cost incurred by using the m-learning platform; the performance cost $R[(I I(\pi)-W)]$ is the time charge of the teacher, where $\mathrm{II}(\pi)$ is the total gain brought by $\mathrm{m}$-learning and $\mathrm{R}$ is the extraction function of the income, i.e., the function of A's teaching quality. B also receives value-added benefit of accepting m-learning: $(1-R)(I I(\pi)-W)$.

In m-learning, A can choose to supervise or not supervise student learning. The two choices are referred to as supervision and non-supervision, respectively. If A selects the supervision mode, he/she has to pay a certain amount of supervision cost C. B can either coordinate or conflict with the teacher. The two behaviours are referred to as coordination and conflict, respectively.

The provider of the m-learning platform serves as the service provider and carrier for the implementation of $\mathrm{m}$-learning. Here, the service capacity coefficient of the platform is denoted as $\xi$. The service capacity depends on many factors, including but not limited to the management capacity, hardware and software, awareness, brand power and penetration rate of the platform.

If $B$ cooperates actively in the course of learning, the students will have a value-added effect on the $\mathrm{m}$ learning quality. The added value is denoted as $\mathrm{V}_{1}$. If $\mathrm{B}$ conflicts with the teacher, the students will cause impairment of value. The value loss is denoted as $\mathrm{V}_{2}$. For the teacher $\mathrm{A}$, he/she will receive a profit increase $\xi \mathrm{V}_{1}$ by the enhanced quality of the m-learning platform if $\mathrm{B}$ chooses cooperation; otherwise, he/she will suffer from a profit reduction $\xi \mathrm{V}_{2}$.

Here, it is assumed that both value increase and reduction appear during the m-learning, and the ratio of the teacher to students is $\mathrm{R}$ and $1-\mathrm{R}$ in the two cases.

It is also very important to evaluate the satisfaction with the m-learning platform. High satisfaction helps to boost the teacher's development and income, and the retention of students. Based on the above income model, the author introduced the evaluation factors of the m-learning platform. If the platform finds that the teaching quality and student feedbacks are not as expected, it will punish the teacher A. The amount of penalty is denoted as T. Hence, the teacher A should consider both the cost of supervision and the punishment of the platform.

\section{Model building}

In the course of m-learning, if B actively cooperates according to the learning protocol, and pays the degree of effort $\pi_{1}$, then the overall revenue of the m-learning is $\mathrm{II}\left(\pi_{1}\right)$.In this case, theincome of $\mathrm{B}$ is $\mathrm{W}+$ 
$\mathrm{R}\left[\mathrm{II}\left(\pi_{1}\right)-\mathrm{W}+\xi \mathrm{V}_{1}\right]=W(1-R)+\mathrm{R}\left[\mathrm{II}\left(\pi_{1}\right)+\xi \mathrm{V}_{1}\right]$.If $\mathrm{A}$ chooses supervision, his/her income is $(1-$ $R)\left[\mathrm{II}\left(\pi_{1}\right)-\mathrm{W}+\xi \mathrm{V}_{1}\right]-C$. If A chooses non-supervision, the income is $(1-R)\left[\mathrm{II}\left(\pi_{1}\right)-\mathrm{W}+\xi \mathrm{V}_{1}\right]$.

If $\mathrm{B}$ conflicts with the learning protocol, and pays the degree of effort $\pi 2$, then the overall revenue of the $\mathrm{m}$ learning is II $\left(\pi_{2}\right)$, in which B will receive some private interest $\mu$. In this case, the quality of m-learning service will affect the student learning experience. Thus, the platform will punish the teacher A with a penalty $\mathrm{T}$.

If $\mathrm{A}$ chooses non-supervision, he/she will be punished with $\mathrm{T}$ by the platform due to the conflict of $\mathrm{B}$. However, B will not receive any punishment but get extra private interest.At this point, the income of B is $\mathrm{W}+$ $\mathrm{R}\left[\mathrm{II}\left(\pi_{2}\right)-\mathrm{W}+\xi \mathrm{V}_{2}\right]+\mu$, and that of $\mathrm{A}$ is $(1-R)\left[\mathrm{II}\left(\pi_{2}\right)-\mathrm{W}-\xi \mathrm{V}_{2}\right]-T$.

If $A$ chooses supervision, the probability that $A$ notices the conflict of $B$ is $\lambda$, and the frequency of punishment on B is $\delta$. Hence, the probability that B's conflict goes unnoticed is 1- $\lambda$ under the supervision of A. If so, A will be punished by the platform with is $(1-\lambda) \mathrm{T}$. At this point, the income of $\mathrm{B}$ is $\mathrm{W}+$ $\mathrm{R}\left[\mathrm{II}\left(\pi_{2}\right)-\mathrm{W}-\xi \mathrm{V}_{2}\right]+\mu-\lambda \delta$, and that of $\mathrm{A}$ is $(1-R)\left[\mathrm{II}\left(\pi_{2}\right)-\mathrm{W}-\xi \mathrm{V}_{2}\right]-C+\lambda \delta-(1-\lambda) T$.

In this way, a game income matrix was established for the teacher-student interaction in m-learning (Figure 1). In each quadrant, the first row refers to B's income, and the second row stands for A's income.

\section{Teacher $\cdot \mathrm{A}$}

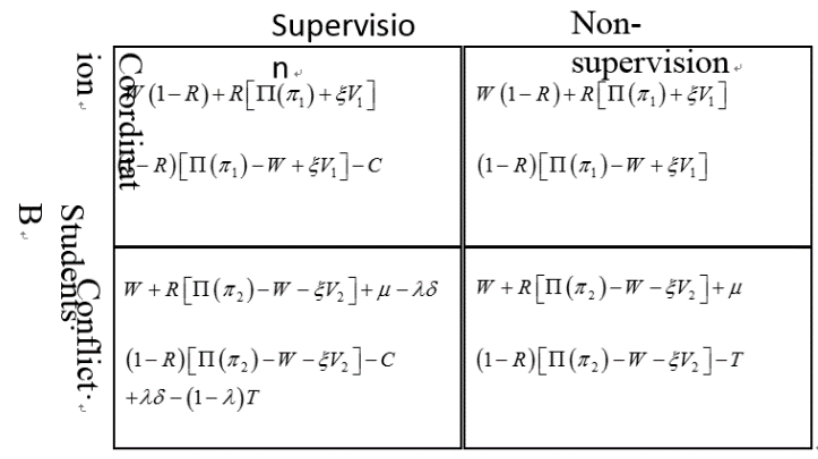

Figure 1. Game income matrix for the teacher-student interaction in m-learning.

Let the probability that A supervises the learning of $\mathrm{B}$ be $\mathrm{X}$, and the probability of non-supervision be 1-X. Suppose the probability that $\mathrm{B}$ chooses cooperation is $\mathrm{Y}$, and the probability of conflict is 1-Y.Thus, the income function of A can be expressed as:

$$
\begin{aligned}
& U_{A}(X, Y)=X\left\{Y\left[(1-R)\left(\Pi\left(\pi_{1}\right)-W+\xi I_{1}\right)-C\right]+(1-Y)\left[(1-R)\left(\Pi\left(\pi_{2}\right)-W-\xi I_{2}\right)-C+\lambda \delta-(1-P) T\right]\right\} \\
& +(1-X)\left\{Y\left[(1-R)\left(\Pi\left(\pi_{1}\right)-W+\xi I_{1}\right)\right]+(1-Y)\left[(1-R)\left(\Pi\left(\pi_{2}\right)-W-\xi I_{2}\right)-T\right]\right\}
\end{aligned}
$$

Find the partial derivative for $\mathrm{X}$ : 


$$
\frac{\partial U_{A}(X, Y)}{\partial X}=-C+(1-Y) \lambda(\delta+T)
$$

Solve $\mathrm{Y}$ at formula $(2)=0$ :

$$
Y=1-\frac{C}{\lambda(\delta+T)}
$$

The income function of B can be expressed as:

$$
\begin{aligned}
& U_{B}(X, Y)=Y\left\{X\left[W(1-R)+R\left(\Pi\left(\pi_{1}\right)+\xi I_{1}\right)\right]+(1-X)\left[W(1-R)+R\left(\Pi\left(\pi_{1}\right)+\xi I_{1}\right)\right]\right\} \\
& +(1-Y)\left\{X\left[W+R\left(\Pi\left(\pi_{2}\right)-W-\xi I_{2}\right)+\mu-\lambda \delta\right]+(1-X)\left[W+R\left(\Pi\left(\pi_{2}\right)-W-\xi I_{2}\right)+\mu\right]\right\}
\end{aligned}
$$

Find the partial derivative for $\mathrm{Y}$ :

$$
\frac{\partial U_{B}(X, Y)}{\partial Y}=R\left[\Pi\left(\pi_{1}\right)-\Pi\left(\pi_{2}\right)+\xi I_{1}+\xi I_{2}\right]-\mu+\lambda \delta
$$

Solve $\mathrm{X}$ at formula $(4)=0$ :

$$
X=\frac{\mu+R\left[\Pi\left(\pi_{1}\right)-\Pi\left(\pi_{2}\right)\right]}{\lambda \delta}-\frac{R\left(\xi I_{1}+\xi I_{2}\right)}{\lambda \delta}
$$

Thus, the Nash equilibrium solution can be obtained:

$$
\left(X^{\prime}, Y^{\prime}\right)=\left\{\frac{\mu+R\left[\Pi\left(\pi_{1}\right)-\Pi\left(\pi_{2}\right)\right]}{\lambda \delta}-\frac{R\left(\xi I_{1}+\xi I_{2}\right)}{\lambda \delta}, 1-\frac{C}{\lambda(\delta+T)}\right\}
$$

\section{Results and Analysis}

Through the analysis of the established model, the author put forward the following suggestions on improving the teacher-student interaction in m-learning:

(1)In m-learning, the service capacity coefficient $\xi$ of the m-learning platform is negatively correlated with the probability X for A to supervise B. In other words, the greater the coefficient, the less likely for the teacher to supervise students. Therefore, the teacher should adopt platforms of strong service capacity to enhance the interaction with students. Moreover, the probability that the teacher chooses supervision is negatively correlated with the value increase $\xi \mathrm{V}_{1}$ and value reduction $\xi \mathrm{V}_{2}$ in m-learning. This means the teacher should also keep track of the value increase/reduction of m-learning platform services, and select the optimal supervision level.

(2) The supervision cost $\mathrm{C}$ of $\mathrm{A}$ over $\mathrm{B}$ is negatively correlated with the probability y for active cooperation with $\mathrm{B}$. This is because $\mathrm{B}$ knows that the higher the $\mathrm{C}$, the less likely for A to choose supervision. With this mindset, B will focus more on their own interest and refuse to cooperate, which dampens the chance of active cooperation. To enhance the teacher-student interaction in m-learning, it is necessary to suppress the egoistic behaviours resulted from information asymmetry, and encourage A and B to interact with each other; the students should enhance their autonomous learning ability, thus reducing the supervision cost of the teacher. 
(3) The private interest $\mu$ of B is positively correlated with the probability for A to choose supervision. In m-learning, the teacher needs to impart and disclose more information, and investigate the students' private interest. In light of the size of the private interest, the teacher should determine the proper supervision probability. In this way, the teacher can reduce unnecessary supervision, lower the supervision cost, and promote the interaction with students.

(4) With the increase in the evaluation factors, the probability Y that B chooses cooperation is positively correlated with the amount of penalty $\mathrm{T}$ imposed by the platform on A. When B cooperates with A or follow the protocol, the service is more likely to reach the expected quality of the platform, pushing down the chance of negative evaluation.

(5) The probability $\lambda$ that teacher A found the conflict of B is negatively correlated with the probability $\mathrm{X}$ for A to supervise B, and positively with the probability $\mathrm{Y}$ that $\mathrm{B}$ chooses cooperation. The amount of penalty $\mathrm{T}$ is negatively correlated with the probability $\mathrm{X}$ for A to supervise $\mathrm{B}$, but positively with the probability $\mathrm{Y}$ that B chooses cooperation. Therefore, the teacher should probe deep into the conflict behaviours of the students. The amount of penalty should be configured with the aim to stimulate student cooperation and reduce teacher's supervision cost.

\section{References}

Bernstein-Yamashiro, B., \& Noam, G. G. (2013). Teacher-student relationships: A growing field of study. New Directions for Youth Development, 13(7), 15-26. http://dx.doi. org/10.1002/yd.20045

Liu, L. J., Xiong, C. P., \& Lin, L. (2011). The experimental research on using SMS interactive platform into mobile teaching. Modern Distance Education, (3), 68-72.

Ma, Z. G., Xu, K. W., \& Tian, F. M. (2010). Internet-based teacher-student relationship: Viewing from "you and I" relationship. Education Science, 26(3), 27-31.

Rutter, M., \& Maughan, B. (2002). School effectiveness findings 1979-2002. Journal of School Psychology, 40(6), 451-475. http://dx.doi. org/10.1016/S0022-4405(02)00124-3

Wan, L. U., Zhao, C. L., Liao, W. W., \& Shu, F. F. (2012). Research on social interaction of e-learning community based on QQ gourp, E-education Research, (9), 54-58.

Zhang, Y. H., \& Zuo B. (2013) Research on the influence factors of the interaction between teachers and students in the Internet. //Abstract of the Symposium on Chinese Psychologists' Symposium, College of Psychology, Beijing Normal University, 1. 